\title{
Hybrid Imaging
}

National Cancer Institute

\section{Source}

National Cancer Institute. Hybrid Imaging. NCI Thesaurus. Code C94909.

Use of a common bed for 2 imaging modalities implemented at or near the same time. 